\title{
Síntesis de nitruro de titanio mediante láser y energía solar concentrada
}

\author{
I. $\operatorname{García~}^{(*)}$, J. Sánchez-Olías ${ }^{(*)}$, J.J. de Damborenea ${ }^{(*)}$ y A.J. Vázquez ${ }^{(*)}$
}

\begin{abstract}
Resumen Se presenta la utilización de la energía solar concentrada mediante lentes de Fresnel para la síntesis de materiales por reacción gas-sólido. Estos primeros resultados sobre nitruración superficial de titanio y aleaciones de titanio se comparan con los obtenidos con técnicas similares como el láser de alta potencia y la lámpara de descarga de xenón. Las capas de nitruro de titanio obtenidas mediante energía solar concentrada por lentes de Fresnel y lámpara de xenón son homogéneas, sin grietas ni defectos, y con un espesor uniforme de $6 \mathrm{~m}$ en tiempos de sólo $2 \mathrm{~min}$. La buena calidad de estas capas se confirma mediante difracción de rayos X.
\end{abstract}

Palabras clave: Nitruro de titanio. Láser. Lámpara de arco. Energía solar.

\section{Titanium nitriding by laser and concentrated solar beams}

\begin{abstract}
The possibility of the employment of solar energy concentrated by Fresnel lens is investigated in order to synthesize materials by gas-solid reaction. These first results are compared by two similar techniques as high power laser and xenon arc lamp. The TiN coatings obtained with xenon arc lamp and Fresnel lens are homogenous, without pores or defects, with a uniform thickness of about $6 \mathrm{~m}$ for treatments of $2 \mathrm{~min}$. The good quality of the TiN coating for all the testing conditions was confirmed by the x-ray diffraction measurements.
\end{abstract}

Keywords: Titanium nitride. Laser. Arc lamp. Solar energy.

\section{INTRODUCCIÓN}

El titanio y sus aleaciones poseen buenas propiedades mecánicas y de estabilidad química unidas a baja densidad, lo que les ha llevado a tener un gran número de aplicaciones. Sin embargo, sus propiedades tribológicas son pobres y un freno a un uso más extendido del titanio. Por este motivo, se han desarrollado distintas alternativas para aumentar su resistencia al desgaste; la mayor parte de éstas están basadas en la creación, por distintas técnicas, de una capa superficial de nitruro de titanio. El nitruro de titanio posee una resistencia al desgaste muy superior a las aleaciones de titanio, a la vez que mejora también las propiedades de resistencia a la corrosión. Las capas de TiN se han logrado obtener por un lado mediante deposición física PVD, química CVD o implantación iónica (1-3) y, por otro lado, mediante métodos térmicos, como son los hornos de inducción (4) o láseres de alta potencia (5-8).

(*) Centro Nacional de Investigaciones Metalúrgicas, CENIM (CSIC), Avda. de Gregorio del Amo 8, 28040-Madrid (España). Tlf.: 34155389 00. Fax: 3415347425 e-mail: igarcia@cenim.csic.es.
Una nueva alternativa a estos métodos consiste en la aplicación de la energía solar, en forma de haz concentrado a altas densidades de energía por distintos tipos de concentradores, al tratamiento de materiales. Como, p.ej., la síntesis de TiN por reacción gas-sólido en la superficie de titanio y aleaciones de titanio. Esta opción posee grandes atractivos y viene siendo explorada, desde hace varios años, por diversos laboratorios (9-15). La energía solar concentrada tiene muchas ventajas evidentes, entre ellas, la gratuidad de una fuente energética que, además, es limpia y no contaminante. Por otro lado, es una técnica con grandes puntos en común con la tecnología láser, con la que puede competir, si se tienen en consideración los costes energéticos, además de que posee ventajas como la debida a trabajar con un espectro que tiene una gran cantidad de energía en las longitudes de onda del ultravioleta y, por tanto, son bien absorbidas por los metales (16) y el TiN (17).

Una etapa intermedia en el uso de la energía solar a la modificación de materiales es la utilización de los llamados simuladores solares, dispositivos concentradores de la energía producida por 
lámparas de descarga de arco como las lámparas de xenón (18-21). Estas lámparas poseen un espectro de radiación muy similar al solar y con una concentración adecuada pueden conseguirse haces con densidades de potencia de $220 \mathrm{~W} / \mathrm{cm}^{2}$. Por consiguiente, los resultados obtenidos son comparables a los que se pueden obtener en instalaciones solares reales.

Todo el trabajo llevado a cabo para la aplicación de la energía solar concentrada a la modificación de materiales se ha hecho mediante instalaciones que concentran la luz solar con espejos parabólicos. La ausencia del uso de sistemas refractivos es lógica si se piensa en lentes convencionales de vidrio, demasiado pesadas y caras cuando se pretenden altas concentraciones.

La alternativa que se propone es el empleo de lentes de Fresnel para la concentración de la luz solar hasta elevadas densidades de energía. En este tipo de lentes, la luz es concentrada por las facetas grabadas con distinto ángulo en uno de los lados de la lente que actúan como prismas que desvían la luz hacia el foco óptico (22). Estas lentes están fabricadas en una fina lámina de material acrílico y son, por tanto, ligeras y baratas.

En este trabajo, se presentan los primeros resultados en la nitruración de titanio usando un nuevo dispositivo de concentración de la energía solar con lentes de Fresnel. Estos resultados se comparan con la nitruración por fusión superficial con láser (23) y con resultados previos obtenidos mediante una lámpara de arco de xenón como simulador solar (24).

\section{DESCRIPCIÓN EXPERIMENTAL}

Se prepararon probetas de titanio y Ti6Al4V de distintas dimensiones para las tres técnicas de modificación empleadas. La superficie expuesta en cada caso a la radiación se rectificó y pulió previamente con papel de granulometría 600 y en el caso del láser se granalló.

Después de cada tratamiento las probetas se cortaron transversalmente y se prepararon para observación metalográfica. La incorporación de nitrógeno se determinó por microscopía óptica y electrónica de barrido y por difracción de rayos de $\mathrm{X}$.

\subsection{Láser}

La fusión superficial en atmósfera de nitrógeno se llevó a cabo con un láser de $\mathrm{CO}_{2}$ de Spectra-Physics operando en modo TEM 20 y con un máximo de potencia de salida de $5 \mathrm{~kW}$ (longitud de onda de 10,6 $\mu \mathrm{m})$. El haz de salida de la cavidad óptica se focalizó a $7 \mathrm{~mm}$, produciendo por tanto un área de trabajo de $40 \mathrm{~mm}^{2}$. La descripción experimental más detallada y las condiciones del proceso se han descrito en un trabajo anterior (23 y 25).

\subsection{Lámpara de arco de xenón}

El dispositivo consta de una lámpara de arco con electrodos de volframio OSRAM XBO y un reflector elíptico. La potencia eléctrica de entrada es de 7 $\mathrm{kW}$ suministrada con un voltaje de $43 \mathrm{~V}$. La densidad de potencia en el foco producido por el concentrador de espejo elíptico se puede incrementar mediante el control de la intensidad de corriente eléctrica aplicada desde $120 \mathrm{~W} / \mathrm{cm}^{2}$ con $100 \mathrm{~A}$ hasta $230 \mathrm{~W} / \mathrm{cm}^{2}$ aplicando $155 \mathrm{~A}$. El arco formado entre los electrodos por la descarga eléctrica en el xenón produce una radiación muy similar al espectro solar entre 300 y $1300 \mathrm{~nm}$ con un máximo de intensidad entre 825 y $900 \mathrm{~nm}$. En un trabajo anterior, se presentó la caracterización de la radiación obtenida con esta lámpara (24).

Las probetas de Ti6Al4V se alojaron en una cavidad hecha en un bloque de alúmina cerrada con un cristal de cuarzo y se sometieron a la radiación concentrada de la lámpara de xenón. En esta cavidad se mantuvo una atmósfera de nitrógeno para producir la reacción superficial gas-sólido en la superficie de las probetas de Ti6Al4V.

\subsection{Lente de Fresnel}

Para la concentración de la energía solar se empleó una lente de Fresnel de 1,2 $\times 0,8 \mathrm{~m}$ con una distancia focal de 1,4 m. La lente está colocada en una montura azimutal para permitir el seguimiento solar. La máxima densidad de potencia obtenida es de $250 \mathrm{~W} / \mathrm{cm}^{2}$ en un foco de $1 \mathrm{~cm}^{2}$.

Las probetas aisladas térmicamente en su lateral por anillos cerámicos se colocaron en una cámara de acero de aproximadamente $500 \mathrm{~cm}^{3}$ y cerrada por un cristal de cuarzo. Como en el caso de la lampara de xenón, el titanio y el Ti6Al4V se nitrura por la reacción gas-sólido de la superficie con el nitrógeno.

\section{RESULTADOS Y DISCUSIÓN}

Todas las probetas tratadas con los parámetros adecuados en cada uno de los métodos presentan el característico color dorado del nitruro de titanio. En la figura 1 se puede observar la superficie de una probeta tratada mediante la lámpara de xenón. Con todos los métodos empleados se incrementó la rugosidad debido al tratamiento.

La microestructura de las muestras preparadas con el tratamiento láser y las preparadas mediante 


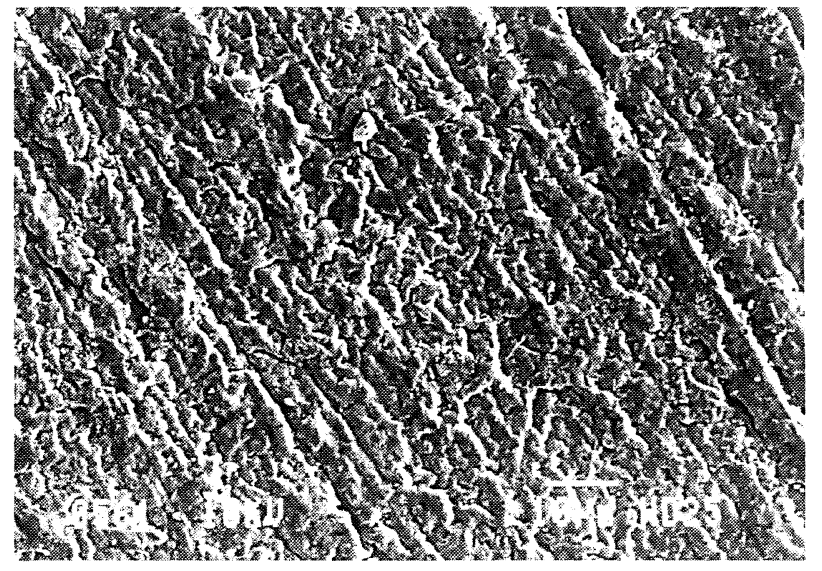

FIG. 1.- Imagen SEM de la superficie de TiN obtenida mediante la lampara de xenón.

FIG. 1.- SEM image of the TiN surface obtained with the xenon arc lamp.

energía solar presentan claras diferencias debido a que, en el primer caso, se ha llegado a la fusión superficial. En la figura 2 se pueden observar las diferentes zonas en una probeta tratada con láser. La primera zona a partir de la superficie, es una capa fina y continua de TiN sobre toda la región fundida. La segunda zona está formada por dendriitas de TiN en una matriz de titanio $\alpha$ enriquecido con nitrógeno. Por debajo de ésta, se puede encontrar una capa de dendritas de TiN sub-estequiométrico en una matriz de titanio. Por debajo de la zona fundida y resolidificada, se puede apreciar, también, la presencia de una zona afectada térmicamente y que ha sufrido una transformación martensítica.

La imagen de SEM de la figura 3 muestra en detalle la capa superficial de TiN, formada por difusión desde la atmósfera de nitrógeno durante la solidificación del cordón laser, que da lugar a una barrera continua sobre la superficie de la probeta.

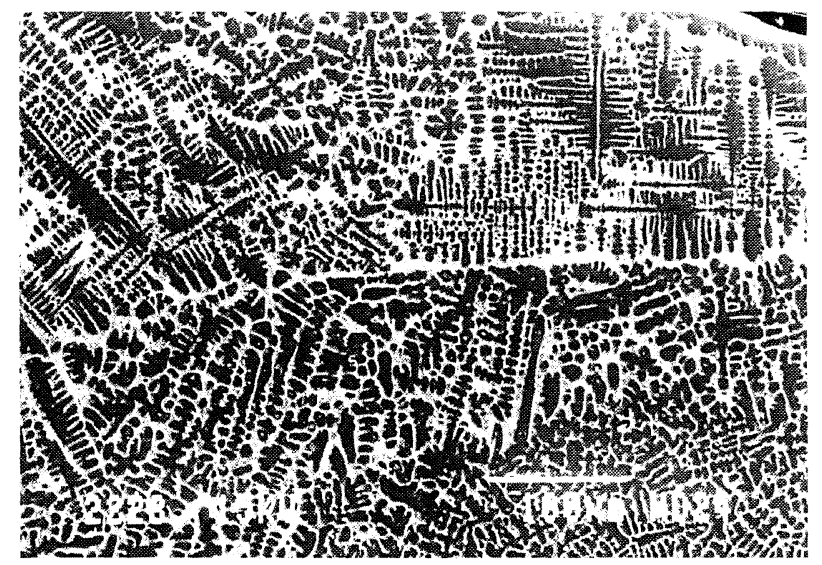

FIG. 2.- Micrografía de la zona afectada por el láser en una probeta de Ti6Al4V. $\times 100$.

FIG. 2.- Micrography of the laser affected zone in a Ti6Al4V specimen. $\times 100$.

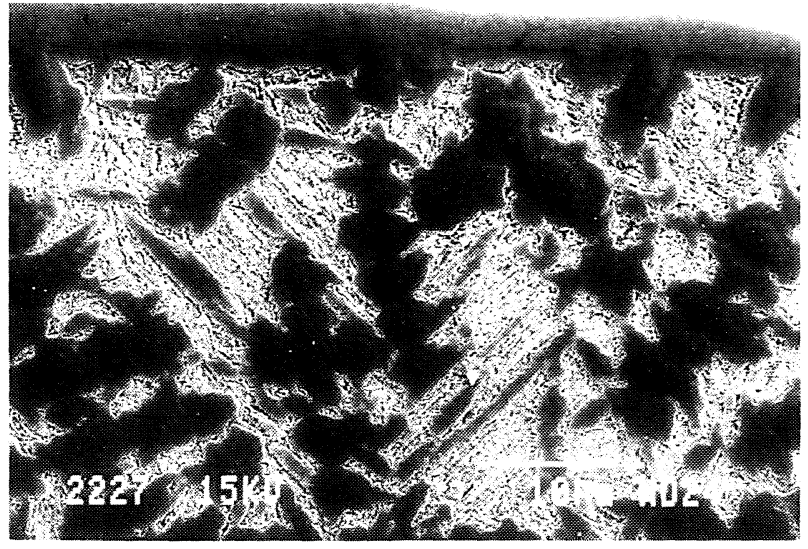

FIG. 3.- Imagen SEM de la capa superficial de TiN en la superficie de una probeta de Ti6Al4V tratada con láser.

FIG. 3. - SEM image of the TiN surface coating in a Ti6Al4V specimen treated by laser.

En el caso de la lámpara de xenón y la lente de Fresnel, la superficie de la probeta no llega a fundirse. Por tanto, como ocurría en la capa más externa en el caso del láser, la capa de TiN crece por la difusión del nitrógeno en la fase sólida. En las figuras 4 y 5 se aprecia la uniformidad y la ausencia de grietas o poros. En estas probetas se pueden establecer cuatro zonas desde la superficie: la capa externa de TiN, una zona de Ti $\alpha$ con nitrógeno disuelto no homogénea debida a la difusión preferente en algunos granos, una zona de $\mathrm{Ti} \alpha$ ' martensítico donde no ha llegado nitrógeno y la zona del material no afectada por ninguna transformación térmica.

Puede apreciarse que la capa nitrurada es muy uniforme en toda la superficie perpendicular a la radiación solar, con un espesor de $6 \mu \mathrm{m}$. Esto significa unas velocidades de crecimiento de

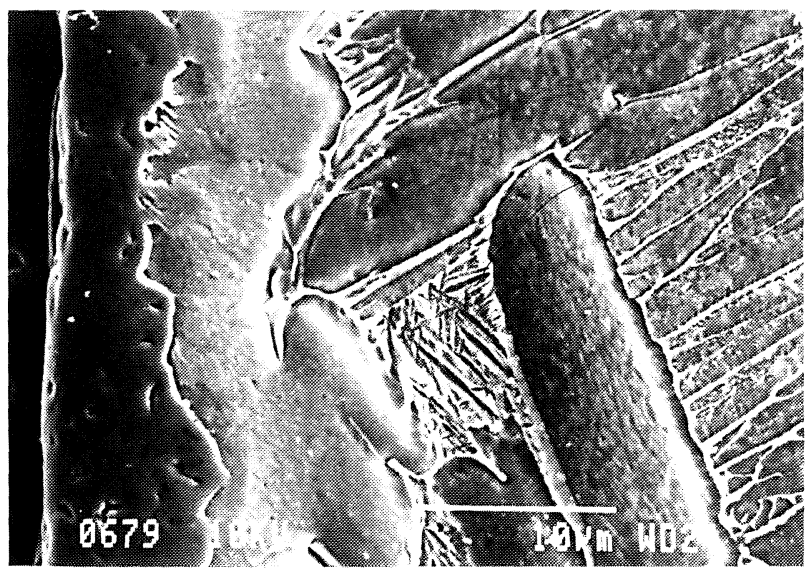

FIG. 4.- Imagen SEM del corte transversal de la superficie de una probeta tratada con la lámpara de xenón durante $40 \mathrm{~s}$.

FIG. 4.- SEM image of the surface of a Ti6Al4V specimen treated with the arc xenon lamp during 40 s. 


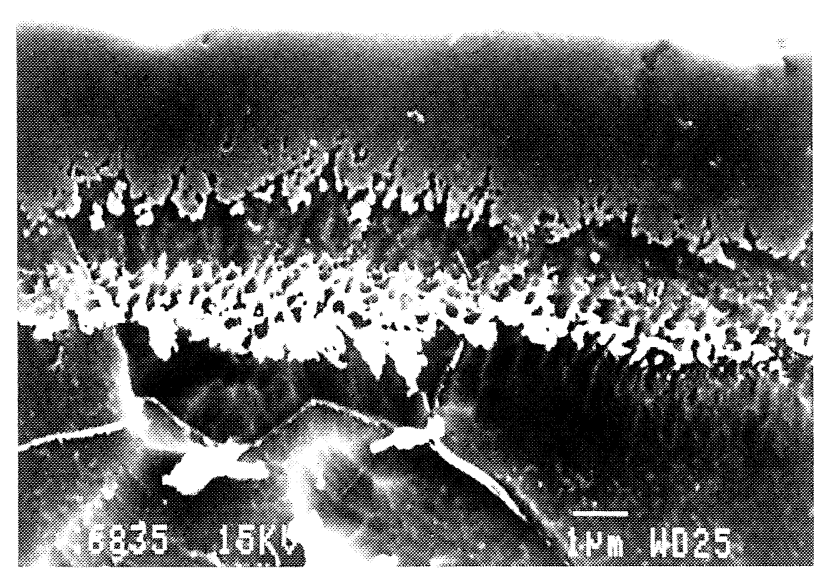

FIG. 5.- Imagen SEM del corte transversal de la superficie de una probeta tratada con energía solar concentrada mediante lentes de Fresnel durante $120 \mathrm{~s}$.

Fig. 5.- SEM image of the surface of a Ti6Al4V specimen treated during 120 s by solar energy concentrated by Fresnel lens.

aproximadamente $180 \mu \mathrm{m} / \mathrm{h}$, mucho mayor que las obtenidas por otros medios más convencionales (1-3), y del mismo orden que las obtenidas en trabajos previos (23 y 25) y por otros autores (5-7).

Para apoyar la discusión metalográfica de las fases, se ha utilizado la difracción de rayos X (figuras 6 y 7). La fase predominante de la capa nitrurada en las probetas tratadas con la lámpara de xenón es TiN, y en el caso de las preparadas con la energía solar concentrada por lentes de Fresnel, $\mathrm{TiN}_{0,3}$ y TiN. La presencia de la fase $\mathrm{TiN}_{0,3}$ se atribuye a una insuficiente incorporación de nitrógeno en la zona intermedia de la capa nitrurada.

\section{CONCLUSIONES}

Se han obtenido recubrimientos de TiN sobre $\mathrm{Ti}$ y Ti6Al4V con diferentes métodos de alta energía,

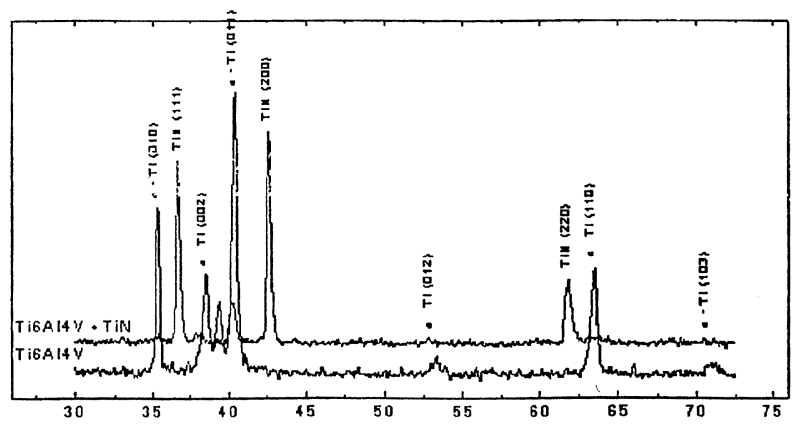

FIG. 6.- Espectro de difracción de rayos X de una probeta de Ti6Al4V antes y después del tratamiento con la lámpara de xenón.

FIG. 6.-X-Ray diffraction spectra of Ti6Al4V before and after a treatment with the arc xenon lamp.

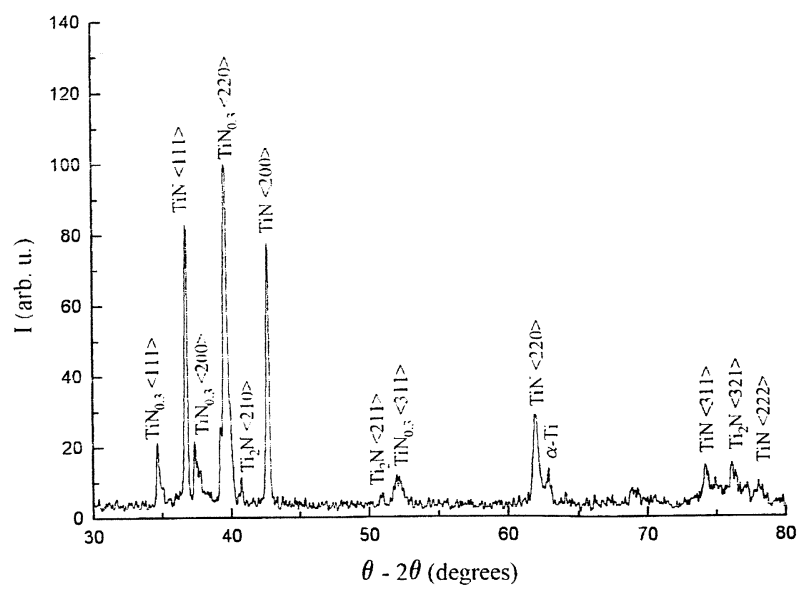

FIG. 7.- Espectro de difracción de rayos $\mathrm{X}$ de una probeta de Ti6Al4V tratada durante 120 s mediante energía solar concentrada por la lente de Fresnel.

FIG. 7.-X-Ray diffraction spectra of Ti6Al4 V treated by solar energy concentrated by Fresnel lens during $120 \mathrm{~s}$.

como láser de $\mathrm{CO}_{2}$, lámpara de arco de xenón y, por primera vez, energía solar concentrada con lentes de Fresnel.

Los recubrimientos obtenidos con la lámpara de xenón como simulador solar y mediante lentes de Fresnel son homogéneos y con una velocidad de crecimiento de $180 \mu \mathrm{m} / \mathrm{h}$, lo que supone tiempos de tratamiento breves en comparación con tratamientos convencionales.

La calidad de los recubrimientos de TiN, como se demuestra con la ayuda de XRD, es muy alta para todos los métodos de tratamiento, con ausencia de óxidos de titanio.

Se ha demostrado la posibilidad de utilizar la energía solar concentrada por lentes de Fresnel para la modificación superficial. Este resultado es muy atractivo debido a que se trata de un proceso de bajo costo y muy ecológico.

\section{Agradecimiento}

Este trabajo ha sido financiado en parte por la UE, a través del proyecto INCO-COPERNICUS ICOP-DEMO-2124-1996, y por la CICYT, a través del proyecto del Plan Nacional de Materiales MAT93-0630-C02-01.

\section{REFERENCIAS}

(1) Aisenberg, S. y Chabot, R. J. Vac. Sci. Technol. 10, 1 (1973) 104.

(2) Telama, A., Torkkell, K., Mantyla, T. y Kettunen, P. J. Mater. Sci. 22 (1987) 2893. 
(3) Williams, A.M. y Buchanan, R.A. Mater. Sci. Eng. 69 (1985) 237.

(4) SÄglitz, M. y Grosch, J. HTM, 49, 5 (1994) 353.

(5) Weerasinghe, V.M., West, D.R.F. y de Damborenea, J.J. J. Mater. Proc. Tech. 58 (1996) 79.

(6) Brown, R., Alias, M.N. y Fontana, R. Surf. Coat. Tech.. 62 (1993) 467.

(7) Reisse, G. y Ebert, R. App. Surf. Sci., 106 (1996) 268.

(8) Milosev, Y. y Navinsek, B. Surf. Coat. Tech. 63 (1994) 173.

(9) Rodriguez, P., Vazquez, A.J. y de Damborenea, J.J. Mater. Sci. Forum. 163-165 (1994) 133.

(10) Pitts, R., Tracy, E., Shinton, Y. y Fields, C. L. Critical Rev. Surf. Chem., 2(4); (1993) 247.

(11) Fernandez, B.J., Lopez, V., VazQuez, A.J. y Martinez, D. ISATA. New y Alternative Materials for Automobile Industries. Florence (1996) 739.

(12) Pitts, J.R., Stanley, J.T. y Fields, C. L. 4th Int Symp. on Res., Dev. Appl. Solar Thermal Tech., Santa Fe, NM, (1988).

(13) Franck, J.P. Celis y Roos, J.R. J. Mater. Res. Vol. 10, No. 1 (1995).

(14) Stanley, J.T., Pitts, J.R. y Fields, C.L. Conf. Proc. 5th Annual Northeast Regional Meeting TMS. Protective Coatings: Processing y Characterisation. Ed. R. R. Yazici. (1989) 43.
(15) Franck, M., Blanpain, B., Oberländer, B.C., Celis, J. P. y Roos, J.R. Solar Energy Mater. Solar Cells, 31 (1993) 401

(16) Vazquez, A.J., Rodriguez, G.P. y De Damborenea, J.J. Solar Energy Mater. 24(1991)731.

(17) Valkonen, E., Ribiing, C.G. y Sundgren, J.E. Appl. Opt. 25 (1986) 3624.

(18) TÖnShoff, H.K. y Meyer-Kobbe, C. Stahl u. Eisen 110 n.4(1990) 157.

(19) Meyer-Kobbe, C. Adv. Mater. Proc. 9(1990)38.

(20) Khun, P. y Hunt, A. Solar Energy Mater. 24(1991)742.

(21) Rodriguez, G.P., Vazquez, A.J. y de Damborenea, J.J. Progress in the Understanding and Prevention of Corrosion, Ed. J.M. Costa y A.D. Mercer, The Institute of Materials, London, vol.2 (1993)1062.

(22) In Solar Cells y Optics for Photovoltaic Concentration. The Adam Higher Series on Optics y Optoelectronics. Adam Higher Ed. (1992).

(23) Garcia, I. y de Damborenea, J.J., pendiente de publicación en Corrosion Science, (1998).

(24) Rodriguez, C., Garcia, I., De Damborenea, J. J. y VAZQuez, A. J. Solar Energy Mater. Solar Cells. 45 (1997) 185.

(25) de Damborenea, J.J., Fernández, B., López, V. y VÁZQuez, A.J. Int. J. Mater. Product. Tech. 11, 3/4 (1996) 301. 